«Une fois découvert l'instrument naturellement adapté à chaque ouvrage, il faut le traduire dans la matière dont on fait l'instrument fabriqué, non pas tel que l'on veut, mais tel qu'il est par nature. Ainsi, il faut savoir transposer dans le fer la percette qui semble naturellement adaptée à chaque ouvrage. » (Socrate dans Platon. Cratyle. Traduction par Catherine Dalimier. Paris : Flammarion, 1998, p. 83, nous soulignons - G. L.-B.) 


\title{
De Mons à Bergen De l'intraduisibilité des noms propres
}

\author{
J ean-Louis VAXELAIRE \\ INALCO, Paris \\ France
}

\begin{abstract}
Résumé : Cet article s'arrête sur la question de l'intraduisibilité des noms propres. Celle-ci naît de l'idée que, puisque le référent est unique, le nom propre qui lui est associé devrait lui aussi être unique et donc intraduisible. Le traducteur ne peut se contenter de ce lieu commun : les noms propres ne peuvent pas être séparés artificiellement du reste du texte et il n'existe pas un traitement simple de la catégorie, certains devant être modifiés, d'autres conservés dans leur forme originale.
\end{abstract}

Mots-clés : noms propres, traduction, normes, sens.

\begin{abstract}
This article tackles the issue of the untranslatability of proper names. Since the referent is unique, the proper name associated with it should also be unique and, therefore, untranslatable. The translator can not be satisfied with this commonplace: proper names cannot be artificially separated from the rest of the text and there is no simple treatment of this category, since some proper names have to be modified, while others are preserved in their original form.
\end{abstract}

Keywords: proper names, translation, norms, meaning.

\section{Introduction}

Dans un article précédent, j'avais choisi en épigraphe une citation de Ionesco qui démontrait que la traduction des noms propres ne posait aucun problème puisque, lorsqu'un Français disait « ma patrie est la France », il suffisait au Portugais de traduire par: «ma patrie est le Portugal». Portugal était alors une traduction parfaite de France.

Par un cheminement inverse, les linguistes (entre autres ReyDebove et Kleiber) ont généralement abouti à la même conclusion en affirmant sans autre forme de procès que les noms propres étaient intraduisibles, les exemples classiques tels que London/Londres et William/Guillaume sont alors considérés comme étant uniquement des adaptations. De nombreux cas sont effectivement des adaptations mais on oublie alors d'expliquer en quoi États-Unis ne serait pas une traduction de United States. La définition du nom propre qu'adopte Mańczak (1968) est 
même fondée sur l'intraduisibilité : le nom propre serait en quelque sorte cet élément qui traverse les langues sans être modifié.

Pourtant, puisqu'il existe des ouvrages de qualité entièrement consacrés à la traduction des noms propres (Ballard 2001, Grass 2002, Podeur 1999), on comprend que l'humour de Ionesco et l'irréflexion des linguistes ne retranscrivent pas la complexité de la question.

Notre approche de la traduction des noms propres est paralysée par un élément, récurrent dans les débats sur les noms propres en général : l'omniprésence du référent. Divers travaux de linguistique ou de philosophie du langage ont finalement perdu de vue que leur matériau est lexical avant d'être physique et mondain. Un des arguments des partisans de l'intraduisibilité des noms propres est ainsi que le référent n'a pas d'équivalent (Ballard 2001, 17). Certes, mais personne n'a jamais tenté de traduire un référent, on ne traduit que des textes. Il est possible que certains noms propres posent des problèmes de traduction parce que l'on ne connaît pas leur référent, mais en conclure qu'ils sont intraduisibles à cause de l'unicité de ce référent relève d'une grande naïveté.

Les problèmes posés par la traduction des noms propres sont donc bien plus délicats qu'on ne le pense généralement. La première raison en est liée au fait que les noms propres sont abordés comme étant une partie du discours à part, ne relevant pas forcément de la langue. Ensuite, on oublie souvent, en parlant de traduction, que la pratique est corsetée par diverses normes liées aux sociétés et par différents critères qui découlent du type de nom propre, du genre textuel, etc. La question du sens des noms propres ne peut être mise sous silence puisqu'elle est sujette à polémique depuis plusieurs siècles et nous terminerons en observant la situation dans quelques exemples de fiction.

\section{Une partie du discours à part}

Dès que l'on aborde la question des noms propres, on est immédiatement confronté à leur statut particulier dans nos sociétés. Il suffit d'ouvrir les grammaires pour s'apercevoir en premier lieu qu'ils ne bénéficient pas du même traitement que les autres parties du discours et, en second lieu, qu'ils sont le plus souvent réduits à deux catégories (les anthroponymes et les toponymes) alors qu'il existe bien plus d'une dizaine de types de noms propres dans certaines typologies (Zabeeh 1968, Grass 2001, Vaxelaire 2005). Les noms propres sont considérés comme étant si différents qu'ils en sont parfois oubliés ou exclus. En lexicographie française, l'absence des noms propres dans la nomenclature des dictionnaires de langue est un dogme presqueinviolable.

Un rapport du Conseil supérieur de la langue française (publié au J ournal officiel du 6 décembre 1990) consacré aux emprunts explique qu'il 
faut supprimer les signes diacritiques qui n'existent pas en français (par exemple dans les mots qui contiennent $\tilde{n}$ et å) mais que ceux-ci « subsisteront dans les noms propres seulement ». Le Conseil estime donc qu'il est possible de rapprocher de la graphie française les noms communs ou les adjectifs mais pas les noms propres. Aucune justification n'est donnée, ce choix théorique semble si évident qu'il n'est pas utile de l'expliciter. Il s'agit pourtant d'une décision qui n'est pas naturelle, aucune loi ne nous obligeant à partiellement franciser les noms communs empruntés ou, inversement, à ne pas graphiquement franciser les noms propres étrangers.

Globalement, on agit comme si les noms propres étaient des éléments extra-linguistiques, il semblerait qu'il n'est pas utile d'étudier leur comportement syntaxique, leur orthographe ${ }^{1}$ et leur sémantisme. On se comporte donc comme s'ils ne faisaient pas réellement partie du texte. Nous sommes ici confrontés au nœud du problème pour le traducteur : le matériau sur lequel ce dernier travaille est justement le texte. Si celui-ci contient des noms propres, ce qui est fort probable, le traducteur ne peut faire comme s'ils n'existaient pas puisque ce sont des éléments de ce texte. Une certaine ligne linguistique (Noailly 1999, par exemple) évacue le problème en déclarant le nom propre hors langue et, par conséquent, hors texte. Le nom propre n'est alors plus un problème pour le traducteur puisqu'il n'est généralement pas modifié, à part quelques exceptions recensées par les dictionnaires bilingues. L'approche de la traduction automatique est équivalente. Lors de la première année qui a suivi l'élection de George Bush, le logiciel Reverso traduisait son nom par Georges Buisson. Après quelques réclamations, le nom avait été intégré à la base de données et demeurait alors George Bush dans les traductions vers le français. La manière de traiter les noms propres est souvent extrêmement simple: ce qui est repéré en tant que nom propre, c'est-à-dire comme élément comportant des majuscules bien que n'étant pas en début de phrase, est conservé à l'exception de ceux qui sont déjà intégrés dans le dictionnaire (du type London =Londres).

Toutefois, cette manière de procéder oublie que la classe des noms propres est extrêmement hétérogène et que tous les types de noms propres ne respectent pas ce schéma de non modification.

\section{Normes}

La volonté de traiter différemment la traduction des noms propres et des autres parties du discours est variable selon les pays: les

\footnotetext{
${ }^{1}$ Un lieu commun très répandu voudrait que les noms propres n'aient pas d'orthographe. On peut douter que les professeurs de français apprécient que leurs élèves citent les œuvres de Ballezaque et de Flobère. De même, il n'existe qu'une graphie possible pour le mont Blanc.
} 
anglophones ont tendance à conserver les formes originales là où les Latins ont plus l'habitude de latiniser les noms étrangers ${ }^{2}$.

Ainsi, certains hispanophones, peut-être parce que la précision prononciative leur semble plus importante que l'orthographe, ont écrit le nom du général de Gaulle avec une graphie hispanisante, ce qu'on peut trouver dans l'exemple Aeropuerto Charles de Gol. Ce choix n'est pas du goût de tout le monde, j'ai ainsi pu lire sur une page de discussion de Wikipedia quelqu'un qui se demandait ironiquement quelle graphie il fallait choisir pour les présidents français :

¿Georges Pompidou o yorch pompidú o jorge pompidú?

¿Valéry Giscard d'Estaing o valerio yiscardestein?

¿François Mitterrand o fransua miteran o Francisco Miteran o Paco?

http:// www.ritmodominicano.com/ wiki.php?title=Discusi\%C3\%B3n:Guec

ho/ Archivo1

Si ces propositions nous font sourire, à l'instar de George Bush devenant Georges Buisson, c'est parce qu'elles s'opposent à notre pratique habituelle qui est de ne pas traduire les noms des personnes, à l'exception des contextes humoristiques.

Cependant, on peut objectivement penser que ces graphies sont possibles, une seule ne l'est pas, il s'agit de Paco pour François Mitterrand ${ }^{3}$. S'il n'est pas possible d'opter pour Paco, c'est en raison des normes de nos sociétés occidentales: le président d'un pays doit être respecté et l'usage de son prénom et, a fortiori, d'un diminutif, sera rejetét.

Nul ne peut nier que les choix de traduction sont obligatoirement liés aux normes de la société d'accueil. Toutefois, ces normes ont pour particularité d'évoluer dans le temps. Ainsi, si j'estime que les graphies Fransua Miteran et Francisco Miteran sont envisageables, c'est parce que

2 Comme je l'ai démontré ailleurs (Vaxelaire 2006), il faut nécessairement prendre en compte la langue source: on traduira bien plus souvent en français les noms chinois ou danois que les noms anglais ou espagnols. La proximité géographique et culturelle est un critère essentiel dans le choix de modifier ou non les noms propres étrangers en français. Personne ne songerait en France à traduire Trafalgar Square en place de Trafalgar alors que cela ne choque personne qu'il existe une place Tienanmen à Pékin (ou Beijing) et une place Rouge à Moscou. De même, lorsque Rey rejette les «noms hybrides comme Mme Arrowsmith et Mle McLaren » $(1973,283)$, il oublie simplement qu'on n'utilise aucune forme appellative originale avec les noms grecs ou suédois par exemple: les versions françaises ne regorgent pas de Kyria Antoniou ou de Ms J onsson mais emploient plutôt des Madame Antoniou et MadameJ onsson.

${ }^{3}$ Paco est imaginable dans un contexte particulier tel qu'un dessin humoristique, mais pas en tant que traduction autorisée et officielle.

${ }^{4}$ Ce qui explique que le choix des Américains d'appeler l'ancien président irakien Hussein par son prénom, Saddam, ait autant surpris. Il faut croire que l'ennemi a droit à moins d'égards. 
cela s'est pratiqué lors des siècles précédents. Lorsque l'on lit les Mémoires de La Rochefoucauld, on y apprend que George Villiers est le duc de Bouquinquan. Il ne s'agit pas d'un quelconque village français, mais d'une francisation de Buckingham aujourd'hui disparue. À une époque où le riding coat allait devenir une redingote et le bowling green un boulingrin, rien n'empêchait de franciser des noms étrangers imprononçables pour les locuteurs francophones. Au siècle précédent, on lit dans Les mémoires de Martin Du Bellay (1545) que lî̂le de Huicht fait face à Porsemue. Il est, me semble-t-il, quasiment impossible pour un Français contemporain d'y retrouver Wight et Portsmouth. Les usages évoluent rapidement car, dans l'édition récente des Récits de voyage de Morand (Laffont 2001), qui ne datent pourtant que de la première partie du $\mathrm{XX}^{\mathrm{e}}$ siècle, les graphies des noms propres ont été généralement adaptées, seules celles, explique la préface, «qui exhalent un parfum d'époque » (Stamboul, Kiew) ont été maintenues.

En anglais, deux villes portent le même nom de London, une située en Grande-Bretagne, l'autre au Canada, mais une seule possède une version française. L'argument de la fréquence qu'invoque Ballard (2001: 29), et qui expliquerait pourquoi London en Ontario n'est pas traduit, est faux. Ce nom est très souvent employé par les Canadiens francophones, peut-être même plus chez certains que celui de la capitale anglaise, et ils ne le transforment pourtant pas en Londres. L'argument historique me parait plus valable car il suffit de comparer les noms des villes créées en Amérique pour s'en rendre compte : Cordoba en Argentine, Venice en Californie ou Toledo en Ohio ne se transforment pas non plus en Cordoue, Venise ou Tolède. La pratique de francisation des noms de villes étrangères avait déjà disparu lorsque ces villes ont été fondées ou connues par les francophones.

Puisque la tendance actuelle est de conserver les formes originales ${ }^{5}$, les formes francisées les moins courantes disparaissent petit à petit. Ainsi, Cassovie, la version française de Košice, est ignorée par le Petit Robert alors que les versions allemande (Kaschau) et hongroise (Kassa) apparaissent dans l'article. Tout comme Cassovie, les francophones semblent abandonner certaines formes comme Brousse pour la ville de Bursa en Turquie ou Presbourg pour la capitale slovaque Bratislava. Parallèlement à ces abandons de formes francisées, on voit disparaître les accents ajoutés en français (la ville néerlandaise de Breda ne s'écrit plus Bréda comme dans le tableau de Vélasquez La Reddition de Bréda) et, inversement, les signes diacritiques originaux tendent à être rendus en français (Málaga et non plus Malaga dans le Petit Robert des noms propres).

\footnotetext{
${ }^{5} \mathrm{Du}$ moins graphiquement car il est loin d'être certain que les prononciations françaises actuelles de Portsmouth et Wight soient identiques à celles des anglophones.
} 
Toutefois, cette tendance, comme toutes les tendances, n'est pas appelée à demeurer éternellement. Rien n'indique que, lors du prochain siècle par exemple, nous ne reviendrons pas à cette possibilité de franciser la graphie des noms étrangers. Un peu de recul historique ou une vision moins ethnocentrée établit que l'intraductibilité des noms propres est surtout valable dans une perspective anglo-saxonne contemporaine ${ }^{6}$.

Dans God Delusion, Dawkins (2006) relate que les anglophones emploient par une sorte d'euphémisme Nationalists et Loyalists pour parler des heurts en Irlande du Nord plutôt que d'employer les noms Catholics et Protestants. Dans une version française, on peut adopter les formes nationalistes et loyalistes mais aussi utiliser catholiques et protestants car aucune norme n'impose de pratiquer l'euphémisme à ce sujet en France. Il faut sans doute en conclure qu'avant d'être un exercice linguistique, la traduction est une pratique culturelle.

Les noms propres entraînent de nombreuses erreurs de traduction, parfois parce qu'on ne modifie pas un nom qui devrait l'être, d'autres fois en traduisant un nom qui ne l'est jamais, c'est-à-dire en ne respectant pas les normes de la langue cible. Il serait facile de faire un catalogue des erreurs sur des sites comme Wikipedia, celles-ci étant de nature plus large qu'une simple méconnaissance des normes et des habitudes. Pour prendre un exemple, il est possible de traduire les noms d'universités (ce que font beaucoup de chercheurs dans des colloques où ils adaptent le nom de leur institution à la langue du pays où ils se rendent), mais encore faut-il les traduire correctement. Pendant des mois ${ }^{7}$, on a pu lire sur la page française de Wikipedia consacrée à Saul Kripke qu'il était « professeur de philosophie à la cité universitaire de la ville de New York (City University of New York, CUNY) ». On était alors en droit de se demander s'il faisait de la philosophie dans les chambres des étudiants.

\section{Quelques critères qui influent sur la traduction}

Les travaux sur la traduction tendent couramment à se focaliser sur une paire de langues. Bien que ce soit correct du point de vue méthodologique, le risque est alors de tirer des conclusions qui ne fonctionnent que pour ces deux langues. Ainsi, on écrit souvent que les anthroponymes sont employés sans déterminants en prenant l'exemple de l'anglais et du français alors qu'il suffit d'observer le grec ou le portugais pour comprendre que ce critère n'est pas universellement partagé.

\footnotetext{
${ }^{6}$ Il est indéniable que, dans des périodes telles que l'antiquité, où l'étymologie des noms propres est considérée comme essentielle pour appréhender les individus qu'ils désignent, il est utile de traduire ces noms.

7 L'erreur a été corrigée en 2011, il est désormais inscrit qu'il est « professeur de philosophie à l'université de la Ville de New York (CUNY). »
} 
Nous avons déjà vu que la paire de langues en elle-même influencera le choix de modifier ou de conserver le nom propre : il y aura a priori plus de modifications dans le sens chinois-français que pour anglaisfrançais, de même il y en aura plus dans le sens allemand-français que dans le sens allemand-anglais. De nombreux autres critères doivent être pris en compte que nous ne pouvons tous aborder ici (cf. Vaxelaire 2006).

Parmi ceux-ci, le type de nom propre joue un rôle dans le processus de traduction, ce qui explique pourquoi on peut trouver dans la même page (Lessico famigliare, un roman italien de 1963 de Natalia Ginzburg), les deux formes concurrentes J ournal de Genève en français et Ginevra dans la forme italienne puisqu'on ne traduit pas actuellement les titres de journaux. $\mathrm{Au}$ sein de ces catégories de noms propres, on recense également des approches différentes. Par exemple, en regardant la cartographie d'un guide néerlandais sur l'Italie (guide vert Michelin), on peut noter que les noms des villes sont toutes en italien, même lorsqu'il existe des versions néerlandaises (Milaan, Turijn) alors que les autres types de noms sont presque tous traduits : Abruzzen-massief, Aosta-dal, Dolomieten, Golf van Napels, Sicilië, etc.). Dans la plupart des langues, on adapte plus souvent les noms de continents, d'océans, de mers que ceux de villes ou de régions. Ainsi, en français, Great Salt Lake devient le Grand Lac Salé alors que Salt Lake City est conservé dans sa forme originale. Des critères moins objectifs (renommée, habitudes) entrent en ligne de compte. Dans un roman allemand qui se déroule en France (Eine gefährliche Begegnung de Jünger), les nombreux odonymes tel que rue d'Anjou sont conservés en français mais seule la place Stanislas de Nancy est germanisée. À l'inverse, d'autres toponymes de notoriété internationale sont traduits à l'instar du Vieux Port (Alten Hafen) et de la Butte Montmartre(Mühlenberg).

Une fois ces différences de statut établies, le contexte particulier ne doit pas être oublié.

Il est d'usage dans la presse française de traduire les noms de partis politiques ${ }^{8}$. Ainsi, pour des résultats d'une élection italienne, Le Monde (11/04/2006) écrit que l'alliance de centre-droit était composée par : Forza Italia, Alliance Nationale, UDC, Ligue du Nord, Liste Mussolini, Flamme Tricolore, Nouveaux Démo-Chrétiens, Libéraux Italiens, Liste Défense du milieu ambiant, Retraités, "Non à l'Euro ». L'alliance de centre-gauche comprenait: L'Olivier, Refond. Communiste, UDEUR, Communistes Italiens, Fed. Verts, SVP, Italie des Valeurs, Rose au poing. Tous les noms sont donc traduits à l'exception de Forza Italia. Puisque la presse a pris

\footnotetext{
${ }^{8}$ Bien que l'anglais soit présenté comme une langue qui traduit peu les noms propres, on recense des traductions de noms de partis :

«Villepin also faces pressure from inside the ruling Union for a Popular Movement (UMP) headed by Sarkozy, a likely rival for the 2007 presidential race. » (Agence de presse Reuters, 29/03/06).
} 
$l^{\prime}$ habitude de conserver le nom italien du parti de Berlusconi ${ }^{9}$, il aurait sans doute été étrange de le traduire. Toutefois, si l'on change de genre et que l'on lit un article scientifique à l'instar de Dechezelles (2009), les noms de partis sont cette fois-ci employés dans leur forme originale et l'on ne parlera plus de L'Olivier mais de LUUlivo. Il y aurait implicitement un public à qui l'on doit faciliter la tâche (le lecteur de quotidiens) et celui qui peut faire l'effort de lire dans une langue étrangère (le lecteur de revues scientifiques). En observant les textes d'une structure plurilingue telle que la Communauté européenne, on note de nombreux types de noms qui doivent être traduits. Les institutions en font partie: la Cour de Justice Européenne (CJ CE) devient ainsi ang. European Court of Justice (ECJ), all. Europäischer Gerichtshof (EuGH), roum. Curtea Europeană de J ustiţie (CEJ), etc. Il ne faut pas oublier que des contraintes légales peuvent obliger les traducteurs à ne pas conserver certains noms. Il suffit de comparer les situations française et québécoise pour s'en convaincre: les titres de films peuvent être affichés en anglais en France alors qu'en raison de lois plus contraignantes, ils doivent être traduits en français au Québec, ce qui explique que Scary Movie en France soit Film de peur au Québec. Les pays bilingues ou multilingues ne peuvent souvent pas échapper à la traduction : en Belgique par exemple, les villes ont généralement un nom francophone et un néerlandophone qui peut être une adaptation phonologique (Louvain/ Leuven) ou une vraie traduction (Mons/Bergen). Dans la ville de Bruxelles, les panneaux des noms de rues indiquent une version dans chacune des deux langues (rue du Chevreuil/ Reebokstraat).

Enfin, en dehors des contraintes légales, il semble évident que le poids ontologique du nom est le plus souvent pris en compte: on traduit plus souvent et plus facilement les noms de la fiction que les noms du monde réel. Parmi ces derniers, il est plus courant de modifier les surnoms que les toponymes ou les anthroponymes officiels : Ville lumière devient City of Lights en anglais et, inversement, Big Apple est généralement traduit par la Grosse Pomme.

\section{Les sens des noms propres et de leurs dérivés}

Les discussions à propos des noms propres rencontrent un autre problème lié à une confusion entre sens et signification (Vaxelaire 2008). Parce que les noms propres ont pour particularité d'avoir une signification relativement faible ${ }^{10}$, on a souvent considéré qu'ils n'avaient pas de sens.

\footnotetext{
${ }^{9} \mathrm{Il}$ est possible que ce syntagme ait été conservé car il était connu des milieux sportifs. En tout cas, le nom de la dernière incarnation de ce parti est modifié en français : Il Popolo della Libertà devient Le Peuple de la liberté.

10 Les anthroponymes par exemple ne possèdent rarement plus que quelques traits sémantiques : Dupont contient une poignée de sèmes tels que / humain/, / patronyme/ et
} 
Néanmoins, dès qu'ils intègrent un texte, les noms propres, comme toutes les autres catégories du discours, ont un sens, qui peut différer selon les contextes. Pour le traducteur, le constat est simple : ce qu'il doit traduire, ne relève pas de la signification ou de l'étymologie, mais du sens ${ }^{11}$ et les noms propres, ayant par essence un signifié, ne peuvent être ignorés.

Ainsi, si l'on doit traduire cet extrait de journal pour un public de non-spécialistes:

Le porte-parole du gouvernement, François Baroin, est également intervenu, parlant d'« une tribune de la rue de Solférino plutôt que le fruit d'une pensée puissante de diplomates en activité ». (Le Monde, 23/ 02/11)

il serait inutile de traduire littéralement rue de Solférino ou de le laisser tel quel, puisque c'est la métonymie qu'il faut rendre. Dans la logique dominante de non-traduction des noms de rues, il faudrait espérer que le lecteur sache que le siège du parti socialiste se situe dans cette rue, ce qui est peu probable.

Lorsque l'on pratique la traduction, il est rare de ne pas être confrontéà ce type de problèmes, ce que confirme Gentzler :

Proper nouns, for example, have always been troublesome for translators, for they always tend to have a special specific meaning - such as place names that have a special resonance, location, history in the source culture - that invariably gets lost in translation. (Gentzler 1993, 80)

Plusieurs tactiques sont possibles pour pallier ce problème (note de bas de page, remplacement par des équivalents culturels, etc.), aucune n'étant parfaite. La note de bas de page n'est pas réalisable dans les traductions de textes oraux (sous-titrage de films ${ }^{12}$, etc.), rare dans certains genres comme la bande dessinée. Ainsi, dans une BD de Lauzier (citée par Yaguello, 1978), une féministe explique que «C'est l'occasion rêvée de frapper un grand coup ! Des manifestations dans toute la France, la presse, la télé, un bouquin, Gisèle, des occupations d'églises, le paquet, quoi ! ». Dans une traduction, il serait dérisoire de laisser Gisèle sans explication ${ }^{13}$, il faudrait alors ajouter une note de bas de page expliquant qui est Gisèle

\footnotetext{
/ français/ en terme de langue et non de nationalité puisqu'un dénommé Dupont peut être anglais ou belge.

${ }^{11} \mathrm{~J}$ e ne tiens pas compte ici de textes particuliers tels que certains poèmes où le sens s'efface, en partie ou totalement, devant le signifiant.

12 J'ai trouvé un unique exemple dans des sous-titres réalisés par des amateurs d'une série télévisée britannique (Misfits, 2010) où « She's such a chav » devient dans les sous-titres « Elle est tellement chav », accompagné de « expression anglaise pour désigner une fashion victime de mauvais goût, pas très intelligente », ce qui est impossible à lire en temps réel.

13 Le problème dépasse le cadre de la traduction car l'allusion est probablement incompréhensible pour beaucoup de jeunes Français.
} 
Halimi, transposer un nom qui renvoie à une figure du féminisme dans la culture d'arrivée, etc. J e ne souhaite pas dans cet article me prononcer en faveur de tel ou tel procédé, chaque traducteur doit être libre de choisir celui qui lui semble préférable; le point le plus contestable me semble être de conserver des noms étrangers que les lecteurs ne connaissent pas et dont ils ne peuvent percevoir le sens dans le texte qu'ils lisent. Le report devient alors une non-traduction et, par conséquent, un problème pour le lecteur.

Les adjectifs dérivés de noms propres peuvent également provoquer des difficultés. La tactique des dictionnaires est de les considérer comme simplement relatifs, ce qui est faux dans de nombreux cas. En anglais, Google recense ainsi 219 résultats pour la requête « Lynchian jazz » en mai 2011 alors que le réalisateur David Lynch n'est pas un musicien de jazz. Lynchian ne peut être un adjectif relatif, il est incontestablement qualificatif, la traduction par lynchien n'est donc pas obligatoire ${ }^{14}$, le traducteur pourrait par exemple insister sur le caractère étrange et lent de la musique.

\section{Les noms propres de la fiction}

Nous avons noté dans la troisième partie que les noms de la fiction étaient traités différemment des noms du monde réel. Ils sont intéressants pour la question de l'intraduisibilité des noms propres puisque plusieurs d'entre eux sont des contre-exemples à la théorie dominante. Le fait qu'ils soient inventés par un auteur n'est en rien un contre-argument, il ne s'agit pas d'un problème linguistique, ce sont d'aussi bons exemples de noms propres que Paul ou France, à moins de considérer que les noms propres doivent nécessairement renvoyer à des référents réels.

Toutefois, au sein même des traductions des genres de fiction, ces noms occupent une place qui sera obligatoirement distincte: les noms mondains d'un roman subiront un traitement équivalent à celui qu'ils auraient dans d'autres discours, les noms inventés par l'écrivain seront par contre sujets à débat, voire à controverse.

Nombre d'auteurs ont, au fil des siècles, opté pour des noms parlants dans leur langue d'origine. Si elle est en recul depuis plusieurs siècles, cette pratique a été primordiale lors des millénaires précédents et cela dans toutes les civilisations. Les noms des personnages sont alors, comme l'écrit Calame $(1986,160)$ à propos de ceux d'Alcman, Archiloque et Aristophane, des « micro-récits ».

Les tactiques des traducteurs ont depuis été diverses. Pour conserver l'humour d'Aristophane, Debidour a choisi de modifier les noms

${ }^{14}$ Google ne recense d’ailleurs aucune occurrence de « jazz lynchien » ou « jazz lynchéen », les deux adjectifs existant en français. 
de Dikaiopolis, Lamachos, Lysistrata et Cinésias respectivement en J ustinet, Vatenguerre, Démobilisette et Chaulapin. Ces tactiques peuvent également être diverses au sein d'un même texte. Dans une traduction récente des Acharniens (Crémoux 2008), la traductrice a modifié Dikaiopolis en J usteville et Amphithéos en Ambidieu, tout en conservant Lamachos ou Théôros dans leur version originale. Cette approche se retrouve dans la version française du Dialogue des courtisanes de Lucien (1998), où Glycère devient Doucette, Thaïs devient Brillantine, Corinne devient Virginette, Crobyle devient La Touffe, mais Pythias et Lysias par exemple ne sont pas modifiés.

Lors du dernier millénaire, les noms transparents ont progressivement laissé la place à des noms plus neutres, du moins en apparence, qui appelaient moins à la traduction. Toutefois, une tactique assez commune revient à employer des noms qui sont transparents dans une autre langue que celle de l'auteur. Pour prendre un exemple, dans Les Tresseurs de corde de J ean Pliya (1987), le journaliste, militant zélé du régime de terreur en place, s'appelle Ganvivi, ce qui se traduit en fon par «c'est bon le pouvoir». Mais puisque les francophones ne connaissent généralement pas cette langue, on peut supposer que des traducteurs anglais, italiens, etc. conserveraient Ganvivi, le problème se poserait uniquement pour celui qui traduirait en fon.

À notre époque, la traduction des noms de personnages est surtout une question qui se pose pour la littérature enfantine ${ }^{15}$ et les épopées pour (petits et grands) enfants comme Harry Potter ou Le Seigneur des anneaux, dont les noms propres ne sont pas toujours opaques. De plus, comme il est courant dans la littérature enfantine de modifier les noms imprononçables dans la langue cible, plusieurs traducteurs ont choisi de ne pas conserver tous les noms anglais. L'école des sorciers où se rend Harry Potter comprend quatre maisons dont les noms sont plutôt transparents. Les traducteurs de l'espagnol, de l'allemand, du turc et du grec ${ }^{16}$ conservent les noms anglais, alors qu'ils sont naturalisés dans d'autres langues :

\footnotetext{
${ }^{15}$ Dans les aventures de Tintin, le nom du professeur Tournesol est modifié dans toutes les langues auxquelles j'ai eu accès à l'exception du danois. Son nom peut être traduit par un calque (Trifone Tornasole en italien, Trifonius Zonnebloem en néerlandais), subir une adaptation graphique (Turnösol en turc) ou être entièrement modifié (Balduin Bienlein en allemand, Cuthbert Calculus en anglais). Les noms des Dupond/t, qui sont moins transparents sont par contre plus souvent conservés (en danois à nouveau, mais aussi en portugais ou en italien) bien qu'il existe plusieurs adaptations qui suivent le même schéma de deux patronymes paronymes: Schulze und Schultze en allemand, Thomson and Thompson en anglais, Hernández y Fernández en espagnol, Jansen en Janssen en néerlandais, etc.

${ }^{16}$ Le changement d'alphabet amène le traducteur grec à translittérer ces noms mais aussi à

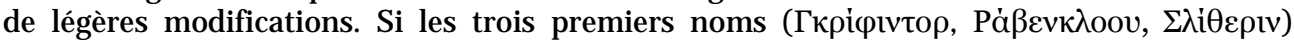
sont le plus proche possible, le dernier (Xá $\varphi \lambda \pi \alpha \varphi \lambda)$ ajoute un -l final qui n'existe pas en
} 


\begin{tabular}{|l|l|l|l|l|l|}
\hline anglais & gallois & tchèque & italien & roumain & finois \\
\hline Slytherin & Slafennog & Zmijozel & Serpeverde & Viperinilor & Luihuinen \\
\hline Gryffindor & Lleureurol & Nebelvír & Grifondoro & Cercetaşilor & Rohkelikko \\
\hline Hufflepuff & Wfftipuff & Mrzimor & Tassorosso & Astropufilor & Puuskupuh \\
\hline Ravenclaw & Crafangfan & Havraspár & Corvonero & $\begin{array}{l}\text { Ochi-de- } \\
\text { Şoim }\end{array}$ & Korpinkynsi \\
\hline
\end{tabular}

Le français entre dans cette catégorie et les quatre maisons de Poudlard (version française de Hogwarts) s'appellent respectivement Serpentard, Gryffondor, Poufsouffle et Serdaigle ${ }^{17}$. Les choix sont assez divers: traduction presque littérale (Griffin en Gryffon), paronymie (Poufsouffle) et modification libre des éléments étymologiques (raven par aigle et slither par serpent).

Le cas du Seigneur des anneaux est distinct car les noms ne sont pas nécessairement aussi transparents et beaucoup sont des créations particulières de Tolkien, relevant de langues imaginaires. Pour aider ses traducteurs, ce dernier a même écrit un Guide to the Names in "The Lord of the Rings" où il indique l'origine des noms, leur signification étymologique et les liens qui les unissent. Pourtant, comme le rapporte Agøy (2003, 32), le traducteur néerlandais déclencha la colère de l'auteur car, selon Tolkien, il détruisait la couleur locale des noms. De même, en français, Ledoux choisit dans sa traduction de conserver le nom Shirriff et de traduire The Shire en la Comté, alors que Tolkien avait indiqué que les deux noms étaient liés.

Le niveau de difficulté est probablement plus important dans Le Seigneur des anneaux que dans Harry Potter car les noms sont moins transparents en anglais. Ferré, Lauzon \& Riggs (2003, 65) prennent l'exemple du toponyme Hushey qui n'est pas modifié dans la traduction de Ledoux : Tolkien juge que ce nom doit être traduit car il renvoie à Rush-isle, ils proposent donc de traduire rush par jonc et d'y ajouter isle, l'ancienne orthographed'île, ce qui aboutit à J oncquisle.

Dans ce type de littérature également, les pratiques se modifient et tendent à conserver les formes originales. Il est intéressant de noter qu'un autre roman de Tolkien, The Hobbit (Bilbo le Hobbit en français) a connu une première traduction en espagnol en Argentine (1964) sous le titre El Hobito, puis une seconde en Espagne (1983) plus proche de l'original : El Hobbit. La situation est encore plus parlante en portugais où la première publication (1962) avait pour titre O Gnomo alors que toutes les traductions suivantes sont devenues O Hobbit.

anglais. De même, la translittération du nom du personnage féminin principal Hermione

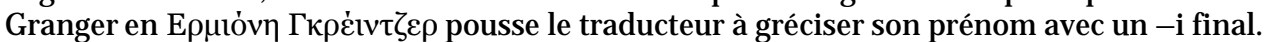

17 Un amateur de jeux vidéo m’a signalé que dans la version localisée en français du jeu Harry Potter, les noms des maisons sont conservés en anglais. 
Les autres genres littéraires regorgent de transformations de noms propres, il serait impossible de tous les citer, je terminerai donc par une poignée d'exemples venant d'horizons très divers et d'époques différentes. Tout d'abord, dans Guzman d'Alfarache d'Alemán (1599-1604), le personnage Pero Crespo $(1968,75)$ est un paysan grossier, mais doué de bon sens et d'équité ; il devient Philippot Colas en français, parce qu'il s'agit d'un nom de vilain dans le vieux folklore français. Le transfert culturel a dû sembler la meilleure solution bien qu'elle soit sujette à caution. En comparant les deux traductions françaises de Max Havelaar de Multatuli (1860), Van Baardewijk-Rességuier (1994 : 108) remarque que les noms des personnages sont tous conservés à l'exception du luitenant Slobkous qui devient le lieutenant Guêtre dans la première traduction de 1968 et le lieutenant Houseaux dans la seconde de 1991. Puisqu'il est le seul à être naturalisé, les lecteurs risquent fort de penser qu'il s'agit d'un personnage français.

On peut aussi penser que des principes d'ordre ontologique entrent en ligne de compte. Dans une traduction récente de Monsieur Prokhartchine de Dostoïevski (1994), on apprend que le nom du héros peut être transparent pour le lecteur russophone puisque gagner ses khartchi, c'est gagner péniblement sa nourriture, son croûton de pain. On voit, page 10, d'autres personnages ayant des noms qui peuvent être découpés: Oplévaniev (du verbe plévat', «cracher »), Prépolovenko (de polovina, «moitié») et Soudbine (de soudba «le destin»), mais le traducteur a pourtant choisi de les conserver dans leur version originale. Pourtant, lors de la page précédente (p. 9), on lit que « cet événement se produisit encore aux Sables, du temps qu'Oustinia Fédorovna n'entretenait que trois clients » : il est certain que ce quartier de Pétersbourg ne s'appelle pas « les Sables » en russe, il a donc été traduit en français. Ce qui n'est pas fait pour le nom de personnage, alors que leur étymologie les rendait potentiellement transformables, l'est donc pour le nom de quartier, ce qui ne choque pas outre mesure les lecteurs. À l'inverse, on imagine mal un quartier de Londres traduit en français et l'on se retrouve à nouveau face au poids de la langue source : le traducteur aura plus de difficultés à manipuler les noms anglais que les noms russes ou grecs. Ce caractère intouchable de l'anglais entraîne parfois des conséquences étonnantes comme dans les sous-titres français d’Un tramway nommé Désir où un personnage annonce : " on m’a dit de prendre un tramway nommé 'Desire', descendre à l'arrêt 'Cemetary' et prendre la rue 'Elysian Fields' ». Étant donné que désir est déjà traduit dans le titre, le choix de le conserver en anglais dans ces sous-titres est pour le moins illogique. 


\section{Pour conclure}

L'idée même de travailler uniquement sur la traductibilité ou l'intraduisibilité des noms propres implique qu'il s'agirait d'une partie du discours bien distincte des autres, il ne viendrait à l'idée de personne de discuter de la traductibilité des adverbes. Une approche textuelle, qui doit nécessairement être celle des traducteurs, indique pourtant qu'il est difficile, voire artificiel, de distinguer les noms propres des autres éléments des textes de langue source. Toutefois, c'est là où se situe la particularité actuelle des noms propres, certaines normes poussent le traducteur à en conserver un nombre plus ou moins important dans leur forme originale. À nouveau, il serait naïf de croire que seuls les noms propres étrangers sont conservés dans les traductions, la lecture de romans contemporains montre que divers noms communs et adjectifs ne sont pas traduits ${ }^{18}$. Les noms communs peuvent, à l'instar des noms propres, des éléments culturels qu'on décide ou non de modifier. Dans sa version de la Bible de 1555, Sébastien Castellion traduisait holocauste par brulage et cène par souper, des choix qui seraient impossibles puisque les emprunts sont aujourd'hui entièrement implantés.

La tendance actuelle de conservation des noms propres étrangers est théoriquement défendable, plusieurs arguments plaident en sa faveur, mais n'est qu'une des approches possibles, pas une règle. A. de Crémoux (2008 : 18) cite P. Thiercy, un des traducteurs d'Aristophane, qui refuse de modifier tous les noms en comparant la situation à celle d'un érudit étranger qui traduirait Boileau en Beraqua ou Trinkwasser. Cette corrélation est tout sauf évidente : bien que nos normes actuelles s'opposent à ces traductions, elles ne sont pas inimaginables dans d'autres sociétés. On peut affirmer que les anthroponymes ne sont pas traduits en français, mais cet extrait de roman nous amène pourtant à relativiser ce principe :

Mon père était le Soleil Blanc, ma mère la Lune Rouge ; je m’appelle Aucun Moment Particulier de la Nuit. Mais vous pouvez mappeler John. (Zameenzad 2005, 285)

Au-delà de l'humour de ce passage, il suffit d'avoir lu des livres sur la conquête de l'Ouest ou regardé des westerns pour s'apercevoir que les noms des chefs indiens étaient toujours traduits en français ou, au pire, en anglais (à l'instar de Sitting Bull, ce qui est toujours plus simple pour un francophone que Tȟath̆ámka Íyotake).

${ }^{18}$ Le vocabulaire employé sur Internet comprend énormément d'anglicismes qui seraient conservés dans une traduction française. $\mathrm{Si}$ un personnage adolescent d'un roman se rend sur des forums d'Internet, il parlera de topic et non de sujet parce que c'est le terme qui est employé par les adolescents français. 
À moins de considérer que notre nom est une partie inaliénable de notre personne, on peut tout à fait imaginer qu'un dénommé Boileau devienne Trinkwasser en Allemagne. De nombreux immigrés ont adapté leur prénom au pays dans lequel ils vivaient ou en ont même changé, une naturalisation du patronyme. Au J apon par exemple, bien qu'aucune loi ne semble l'obliger, il est apprécié que les étrangers qui demandent la nationalité japonaise modifient leur nom. Ainsi, l'homme politique d'origine finlandaise Martti Turunen est devenu Marutei Tsurunen il y a quelques années.

Croire en l'intraduisibilité des noms propres revient à ne pas percevoir l'hétérogénéité de la classe des noms propres qui est loin de se limiter aux seuls anthroponymes et toponymes (les noms de partis politiques ou d'institutions sont le plus souvent traduits), à oublier l'hétérogénéité dans les pratiques traductionnelles selon les époques, les langues, les pays (on n'aborde pas de la même manière un texte danois au XVII e siècle qu'un texte anglais au XXIe siècle), à négliger l'hétérogénéité selon les genres textuels (la littérature enfantine conserve peu de formes étrangères, contrairement aux articles scientifiques) et, enfin, l'interaction entre tous ces éléments.

\section{Références bibliographiques}

***. Romans picaresques espagnols. Paris : Pléiade, 1968.

***. « Pour Guaino, la tribune des diplomates est 'un tract politique' ». Lemonde.fr, AFP (février 2011). [En ligne]. Mis en ligne le 23 février 2011. URL: http:// www.lemonde.fr/ politique/ article/ 2011/ 02/ 23/ pour-guaino-la-tribune-desdiplomates-est-un-tract-politique_1483933_823448.html. (Consulté le 9 juin 2011)

«Rapport du conseil supérieur de la langue française». Journal officiel (6 décembre 1990). [En ligne]. Mise en lignele 3 juillet 2002. URL: http:// www.academie-francaise.fr/langue/ orthographe/plan.html. (Consulté le 9 juin 2011).

Agøy, Nils Ivar. «A Question of Style. On translating The Silmarillion in Norwegian ». In : Th. Honegger (éd.). Tolkien in translation. Zurich-Berne: Walking Tree, 2003 : 31-43.

Ballard, Michel. Le nom propre en traduction. Gap, Paris : Ophrys, 2001.

Calame, Claude. Le récit en Grèce ancienne - Énonciations et représentations de poètes. Paris : Méridiens Klincksieck, 1986.

Crémoux, Anne (de). Les Acharniens d'Aristophane. Villeneuve d'Ascq : Presses Universitaires du Septentrion, 2008.

Dawkins, Richard. God Delusion. Boston : Houghton Mifflin, 2006.

Dechezelles, Stéphanie. "Des vocations intéressées ? Les récits d'engagement des jeunes de Forza Italia à l'aune du modèle rétributif du militantisme». Revue française de science politique $59: 1$ (2009) : 29-50. 
Dostoïevski, Féodore. Monsieur Prokhartchine. Traduction de André Markowicz. Arles : Actes Sud, 1994.

Ferré, Vincent, Daniel, Lauzon, Riggs, David. « Traduire Tolkien en français : On the Translation of J.R.R. Tolkien's Works into French and their Reception in France ». In : Th. Honegger (éd.). Tolkien in translation. Zurich- Berne, Walking Tree, 2003 : 45-68.

Gentzler, Edwin. Contemporary Theories of Translation. Londres-New York : Routledge, 1993.

Ginzburg, Natalia. Lessico famigliare - Note di Dora Cimara. Turin: Giulio Einaudi, 1972 [1963].

Grass, Thierry. Quoi ! Vous voulez traduire « Goethe» ? - Essai sur la traduction des noms propres allemand-français. Berne : Peter Lang, 2002.

Kleiber, Georges. Problèmes de référence: descriptions définies et noms propres. Metz : Centre d'Analyse Syntaxique, 1981.

Mańczak, Witold. «Le nom propre et le nom commun ». Revue Internationale d'Onomastique 20 : 3 (1968) : 205-218.

Noailly, Michèle. «La querelle des noms propres ». Modèles linguistiques 39 : XX (1999) : 107-112.

Pliya, J ean. Les Tresseurs de corde. Paris : Hatier, 1987.

Podeur, Josiane. Nomination in azione - il nome proprio nelle traduzioni dall'italiano al francese e dal francese all'italiano. Naples : Liguori, 1999.

Rey, J ean. Dictionnaire sélectif et commenté des difficultés de la version anglaise. Paris-Gap : Ophrys, 1973.

Rey-Debove, J osette. «Le signe et la chose dans le discours métalinguistique ». Travaux de linguistique et de littérature $7: 2$ (1969) : 113-129.

Samosate, Lucien de. Dialogues des courtisanes. Traduction par Pierre Maréchaux. Paris: Arléa, 1998.

Van Baardewijk-Resseguier, Jacqueline. «Culture et traduction : les références culturelles dans deux traductions françaises du roman néerlandais Max Havelaar ». Linguistica Antverpiensia 27 (1994) : p. 95-112.

Vaxelaire, Jean Louis. Les noms propres - Une analyse lexicologique et historique. Paris : Honoré Champion, 2005.

Vaxelaire, Jean Louis. "Pistes pour une nouvelle approche de la traduction automatique des noms propres ». Meta $51: 4$ (2006) : 719-738.

Vaxelaire, J ean Louis. "Etymologie, signification et sens ». In : J. Durand, B. Habert \& B. Laks (éd.). Actes du Congrès mondial de linguistique française. Paris : EDP, 2008 : 2187-2199.

Yaguello, Marina. Les mots et les femmes. Paris : Payot, 1978.

Zabeeh, Farhang. What's in a Name? - An Inquiry into the Semantics and Pragmatics of Proper Names. La Haye : Martinus Nijhoff, 1968.

Zameenzad, Adam. Pepsi et Maria. Traduction de Brice Matthieussent. Paris : Bourgois, 2005. 